\title{
THRESHOLD DYNAMICS FOR A CLASS OF STOCHASTIC SIRS EPIDEMIC MODELS WITH NONLINEAR INCIDENCE AND MARKOVIAN SWITCHING
}

\author{
A. El Koufi ${ }^{1, *} \odot$, A. Bennar ${ }^{1}$, N. Yousfi $^{1}$ And M. Pitchaimani ${ }^{2}$
}

\begin{abstract}
In this paper, we consider a stochastic SIRS epidemic model with nonlinear incidence and Markovian switching. By using the stochastic calculus background, we establish that the stochastic threshold $\mathcal{R}_{s w t}$ can be used to determine the compartment dynamics of the stochastic system. Some examples and numerical simulations are presented to confirm the theoretical results established in this paper.
\end{abstract}

Mathematics Subject Classification. 34C12, 60H10, 92D25.

Received April 15, 2021. Accepted September 23, 2020.

\section{INTRODUCTION AND PRELIMINARIES}

\subsection{Introduction}

Stochastic calculus generally has been applied in various areas such as economics, physics, biology and epidemiology. Recently, many classes of stochastic epidemic models have been proposed and studied (see for example, $[3,5,8,22,24,30,31,33,35])$. In addition, stochastic models give a real vision, analysis and modelization of infectious diseases compared with the deterministic counterparts [21, 37, 42].

The principal research questions connected with the stochastic modeling and analysis approaches include the study of the existence of a positive solution in the probabilistic sense, the purpose of the threshold, and conditions that guaranteed the persistence or extinction of the disease. Numerous works have been realized in this sense. For example, Zhang et al. [40] introduced the effect of white noise into the deterministic SIQS epidemic model that was suggested by Hethcote et al. [9]. They proved that the system has a unique positive global solution, and presented a threshold of the proposed stochastic system that determines the extinction and persistence of the disease. Zhang et al. [41] proposed a stochastic SIRS epidemic model with media coverage and saturated incidence rate. They discussed the stochastic endemic dynamics by using suitable Lyapunov functions, and the ergodic stationary distribution of stochastic model by using the method of Khasminskii [17].

In mathematical epidemiology, the incidence rate of a disease indicates the number of new infectious cases in the population per unit of time. The most incidence function used in mathematical epidemiological models is

Keywords and phrases: Epidemic model, Markov switching, stochastic threshold, persistence, extinction.

${ }^{1}$ Laboratory of Analysis, Modeling and Simulation (LAMS), Faculty of Sciences Ben M'sik, Hassan II University, P.O. Box 7955 Sidi Othman, Casablanca, Morocco.

2 Ramanujan Institute for Advanced Study in Mathematics, University of Madras, Chennai 600 005, Tamil Nadu, India.

* Corresponding author: elkoufiamine1@gmail.com 
the bilinear incidence rate (simple mass action) $[13,14]$. However, there exist other types of incidence functions and each one represents a specific role (see, $[1,6,12,16,38]$ ). Many scholars illustrate their interest in the study of the effect of nonlinear incidence rate in epidemic models. In [8], Han et al. proposed a stochastic SIR epidemic system with mixed nonlinear incidence rates, they investigated the asymptotic dynamics of the stochastic system by employing Markov semigroup theory and the Fokker-Planck equation and gave sufficient conditions for extinction of disease. Rajasekar et al. [31] proposed a stochastic SIR epidemic model with logistic birth to investigate the threshold of a system that consists of susceptible, infectious and removed individuals. They demonstrated that the extinction and persistence of the disease in their stochastic model are showed by the value of the proposed stochastic basic reproduction number. Also they used the Lyapunov function method to establish sufficient conditions for the existence of a stationary distribution of the positive solutions to the proposed model. In [33], authors investigated a class of stochastic SIS epidemic models with nonlinear incidence and periodic coefficients. In [35], Tang et al. proposed a class of stochastic SIRS epidemic models with nonlinear incidence $\beta f(S) g(I)$ and given the threshold that determines the extinction and persistence in the mean of the epidemic disease. In addition, they showed the existence of a unique stationary distribution of the model under sufficient conditions in the threshold $\tilde{R}_{0}$. In [24], the authors investigated stochastic SIRS epidemic model with logistic growth and general nonlinear incidence rate $p(S, I)$. By constructing a suitable Lyapunov function they established sufficient conditions for the existence and uniqueness of an ergodic stationary distribution of the positive solutions to the stochastic system. In this paper, we consider the following stochastic SIRS epidemic model with general nonlinear incidence $f(S, I, R)$

$$
\left\{\begin{array}{l}
\mathrm{d} S(t)=(A-\mu S-\beta f(S, I, R)+\delta R) \mathrm{d} t-\sigma f(S, I, R) \mathrm{d} M_{B}(t), \\
\mathrm{d} I(t)=(\beta f(S, I, R)-(\mu+\gamma+\alpha) I) \mathrm{d} t+\sigma f(S, I, R) \mathrm{d} M_{B}(t), \\
\mathrm{d} R(t)=(\gamma I-(\mu+\delta) R) \mathrm{d} t,
\end{array}\right.
$$

where $S(t), I(t)$ and $R(t)$ denote the number of susceptible, infected and recovered individuals at time $t$, respectively. $A$ denotes the recruitment rate of the susceptible class, $\mu$ represents the natural death rate of $S$, $I$ and $R$ class, $\gamma$ is the recovery rate of infectious individuals, $\delta$ means that the rate of the loss of immunity for recovered individuals, $\alpha$ is the disease-related death rate of infectious individuals, the transmission of the infection is modeled by a nonlinear incidence rate $\beta f(S, I, R)$, where $\beta$ represents the transmission rate between $S$ and $I$. In addition, $M_{B}(t)$ is the independent standard Brownian motion defined on a complete probability space $\left(\Omega, \mathcal{F},\{\mathcal{F}\}_{t \geq 0}, \mathbb{P}\right)$ with a filtration $\{\mathcal{F}\}_{t \geq 0}$ satisfying the usual conditions, and $\sigma$ is the intensity of stochastic perturbation.

The function $f(S, I, R)$ satisfies the following hypotheses. Let

$$
\Xi=\left\{(S, I, R) \in \mathbb{R}_{+}^{3} \mid S>0, I \geq 0, R \geq 0\right\}
$$

$\left(\mathbf{A s}_{1}\right) f$ is nonnegative and twice continuously differentiable for any $(S, I, R) \in \Xi$, and monotonically increasing with respect to $S$ and monotonically decreasing with respect to $R$.

$\left(\mathbf{A s}_{2}\right) f(S, 0,0)=0$ and $\frac{f(S, I, R)}{I} \leq \frac{\partial f\left(m_{S}, 0,0\right)}{\partial I}$ for all $(S, I, R) \in \Xi$, moreover $\frac{\partial f\left(m_{S}, 0,0\right)}{\partial I}>0$.

The basic reproduction number $R_{0}$ of the corresponding deterministic system of (1) is defined as follows:

$$
R_{0}=\frac{\partial f\left(m_{S}, 0,0\right)}{\partial I} \frac{\beta}{\mu+\gamma+\alpha}
$$

where $m_{S}=\frac{A}{\mu}$. The value of the basic reproduction number $R_{0}$ indicates the extinction or persistence of the disease. It also describes the dynamic behaviors of the deterministic model. 
The stochastic model (1.1) has been addressed by Riffat et al. in [32]. They discussed the asymptotic properties of the solution according to the following stochastic threshold value:

$$
\widetilde{R}_{0}=R_{0}-\left(\frac{\partial f\left(m_{S}, 0,0\right)}{\partial I}\right)^{2} \frac{\sigma^{2}}{2(\mu+\gamma+\alpha)}
$$

Other environmental noise can be introduced in the epidemics models named color noise or telegraph noise [15, 18, 29] and can be illustrated like a switching between two or more regimes of environment which change through factors such as socio-cultural factors, changes in climate and nutrition. Often the switching among different environments is memoryless and the waiting time for the next switching follows an exponential distribution $[2,39]$. Therefore the regime-switching can be modelled by a continuous time Markov chain $\{r(t)\}_{t>0}$ with values in a finite state space $\mathbb{I}=\{1,2, \ldots, N\}$. In [19] Lan et al. explored the dynamics of a stochastic SIRS epidemic model with non-monotone incidence rate and Markovian switching. They addressed the threshold value of the disease that determines persistence and extinction of the disease and using the Markov semigroups theory they proved that the densities of the distributions of the solution can converge in $L^{1}$ to an invariant density. Phu et al. in [28] studied the longtime dynamics of a stochastic SIS epidemic model with general incidence functional response under regime-switching. They attained a sufficient and almost necessary condition for the extinction and persistence of the epidemic system and discussed the rate of all convergence of the solution. Motivated by the above works, in this paper, we propose the following stochastic SIRS epidemic model with both white noise and telegraph noise perturbations and nonlinear incidence:

$$
\left\{\begin{array}{l}
\mathrm{d} S(t)=(A(r(t))-\mu(r(t)) S-\beta(r(t)) f(S, I, R)+\delta(r(t)) R) \mathrm{d} t-\sigma(r(t)) f(S, I, R) \mathrm{d} M_{B}(t), \\
\mathrm{d} I(t)=(\beta(r(t)) f(S, I, R)-(\mu(r(t))+\gamma(r(t))+\alpha(r(t))) I) \mathrm{d} t+\sigma(r(t)) f(S, I, R) \mathrm{d} M_{B}(t), \\
\mathrm{d} R(t)=(\gamma(r(t)) I-(\mu(r(t))+\delta(r(t))) R) \mathrm{d} t,
\end{array}\right.
$$

where $A(i), \mu(i), \beta(i), \delta(i), \gamma(i), \alpha(i)$ and $\sigma(i)$ are all positive constants for any $i \in \mathbb{I}$.

It is important to note that our stochastic system (1.2) includes and improves many stochastic models existing in the literature. For example, the stochastic model with Beddington-DeAngelis incidence presented by Lin et al. [20], when $A(i)=\mu(i)$ and $f(S, I, R)=\beta(i) S I / 1+\alpha_{1}(i) S+\alpha_{2}(i) I$. The stochastic switched model proposed by Han and Zhao in [7], when $A=\mu, \alpha=0$ and the infection transmission process is modeled by the bilinear incidence rate (also called the incidence in mass action). The stochastic system addressed by Tuong et al. [36], when $A=K \mu$ (where $K$ is a carrying capacity) and $f(S, I, R)=f(S, I)$. The stochastic models presented in [33], when the switches between two or more regimes of environment are neglected and $f(S, I, R)=f(S) g(I)$, and in [32], when the regime-switching is not considered.

This work aims at studying the asymptotic properties of the proposed stochastic switched epidemic model (1.2) and at giving a threshold that determines the extinction and the persistence of the disease and which coincides with the threshold of the corresponding deterministic model in absence of white and colored noise. The organization of this paper is as follows: In Section 2, the sufficient conditions on the extinction of the disease in the model (1.2) with probability one are affirmed and proved. In Section 3, the sufficient conditions on the persistence of the disease in the system (1.2) with probability one are stated and proved. In Section 4, numerical simulations are displayed to confirm the theoretical study of this paper.

\subsection{Preliminaries}

Let $\{r(t)\}_{t>0}$ be a right-continuous Markov chain on the probability space $\left(\Omega, \mathcal{F},\{\mathcal{F}\}_{t \geq 0}, \mathbb{P}\right)$ taking values in a finite-state space $i \in \mathbb{I}$ with the generator $\Phi=\left(\phi_{u v}\right)_{1 \leq u, v \leq N}$ given, for $\Delta t>0$, by

$$
\mathbb{P}(r(t+\Delta t)=v \mid r(t)=u)= \begin{cases}\phi_{u v} \Delta t+o(\Delta t) & \text { if } u \neq v \\ 1+\phi_{u u} \Delta t+o(\Delta t) & \text { if } u=v\end{cases}
$$


Here, $\phi_{u v}$ is the transition rate from $u$ to $v$ and $\phi_{u v} \geq 0$ if $u \neq v$, while

$$
\phi_{u u}=-\sum_{u \neq v} \phi_{u v}
$$

Assume Markov chain is irreducible $r(\cdot)$ and independent of the Brownian motion $M_{B}(\cdot)$. Hence, there exists a unique stationary distribution $\pi=\left(\pi_{1}, \ldots, \pi_{N}\right)$ of $r(t)$ such that

$$
\pi \Phi=0
$$

and

$$
\sum_{i=1}^{N} \pi_{i}=1, \pi_{i}>0, i \in \mathbb{I} .
$$

For any vector $l=(l(1), \ldots, l(N))^{T}$, let $\hat{l}=\min _{i \in \mathbb{I}}\{l(i)\}$ and $\check{l}=\max _{i \in \mathbb{I}}\{l(i)\}$.

Let

$$
\Lambda=\left\{(S, I, R) \in \mathbb{R}_{+}^{3}: S+I+R \leq \frac{\check{A}}{\hat{\mu}} \triangleq \overline{m_{S}}\right\}
$$

By using the same technique as in [26], we can easily proved, for $X(0)=(S(0), I(0), R(0)) \in \Lambda$, that there is a unique solution $(S(t), I(t), R(t))$ to system (1.2) on $t \geq 0$, and the solution will remain in $\Lambda$ with probability 1 .

We consider the following stochastic system

$$
\mathrm{d} \zeta(t)=f(t, \zeta(t), r(t)) \mathrm{d} t+g(t, \zeta(t), r(t)) \mathrm{d} B(t),
$$

where $B(t)$ is a d-dimensional standard Wiener processes defined on a complete probability space $\left(\Omega, \mathcal{F},\left\{\mathcal{F}_{t}\right\}_{t \geq 0}, \mathbb{P}\right)$. Denote by $C^{1,2}\left(\mathbb{R}^{d} \times \mathbb{S} ; \mathbb{R}_{+}\right)$the family of all nonnegative functions $\mathcal{H}$ defined on $\mathbb{R}^{d} \times \mathbb{S}$ such that they are continuously twice differentiable in $\zeta$ and one differentiable in $t$. The operator $\mathcal{L}[25]$ associated with (1.3) is defined as follows:

$$
\begin{aligned}
\mathcal{L H}(\zeta, i)= & \mathcal{H}_{t}(t, \zeta, i)+\mathcal{H}_{\zeta}(t, \zeta, i) f(t, \zeta, i)+\frac{1}{2} g^{T}(t, \zeta, i) \mathcal{H}_{\zeta, \zeta}(t, \zeta, i) g(t, \zeta, i) \\
& +\sum_{j \in \mathbb{S}} \phi_{i j} \mathcal{H}(t, \zeta, j),
\end{aligned}
$$

where $\mathcal{H}_{\zeta}$ and $\mathcal{H}_{\zeta, \zeta}$ represents the gradient and Hessian of $\mathcal{H}$, and $T$ is the transpose of a matrix.

Remark 1.1. Under $\left(\mathbf{A s}_{1}\right)$ and $\left(\mathbf{A s}_{2}\right)$, we have

$$
\frac{\partial f(0, I, R)}{\partial I}=\frac{\partial f(0, I, R)}{\partial R}=\frac{\partial f(S, 0, R)}{\partial S}=\frac{\partial f(S, 0, R)}{\partial R}, \text { for all }(S, I, R) \in \Xi .
$$

In addition, $\frac{\partial f(S, 0, R)}{\partial I}$ is nondecreasing with respect to $\mathrm{S}$ and nonincreasing with respect to $\mathrm{R}$.

Lemma 1.2. Suppose that $\left(\boldsymbol{A} s_{1}\right)$ and $\left(\boldsymbol{A} s_{2}\right)$ hold. For all $u>v>0$, define

$$
\mathbb{D}=\left\{(S, I, R) \in \mathbb{R}_{+}^{3} \mid v \leq S+I+R \leq u\right\} .
$$


Then

$$
\begin{gathered}
\max _{(S, I, R) \in \mathbb{D}}\left\{\frac{f(S, I, R)}{S}, \frac{f(S, I, R)}{I}\right\}<\infty, \\
\max _{(S, I, R) \in \mathbb{D}}\left\{\left|\frac{1}{I} \frac{\partial f(S, I, R)}{\partial I}-\frac{f(S, I, R)}{I^{2}}\right|,\left|\frac{1}{I} \frac{\partial f(S, I, R)}{\partial S}\right|\right\}<\infty .
\end{gathered}
$$

\section{ExtinCtion}

In this section, we are interested in the extinction of the disease. Next, we define the threshold of our stochastic system (1.2) by

$$
\mathcal{R}_{s w t}=\frac{\sum_{i=0}^{N} \pi_{i} \beta(i) \frac{\partial f\left(\overline{m_{S}}, 0,0\right)}{\partial I}}{\sum_{i=0}^{N} \pi_{i}\left[\mu(i)+\lambda(i)+\alpha(i)+\frac{\sigma^{2}(i)}{2}\left(\frac{\partial f\left(\overline{m_{S}}, 0,0\right)}{\partial I}\right)^{2}\right]} .
$$

Theorem 2.1. Let $(S(t), I(t), R(t))$ be a solution of the epidemic model (1.2) along with initial value $(S(0), I(0), R(0)) \in \Lambda$. If

$$
\mathcal{R}_{\text {swt }}<1, \quad \text { and } \frac{\partial f\left(\overline{m_{S}}, 0,0\right)}{\partial I} \leq \frac{\beta(i)}{\sigma^{2}(i)} \text { for all } i \in \mathbb{I}
$$

then

$$
\lim _{t \rightarrow \infty} I(t)=0, \quad \lim _{t \rightarrow \infty} R(t)=0 \quad \text { a.s. }
$$

Namely, the epidemic infection dies out from the population with probability one.

Proof. Appling Itô's formula on $\ln I(t)$, we obtain

$$
\begin{aligned}
d \ln I(t)= & {\left[\beta(r) \frac{f(S, I, R)}{I}-(\mu(r)+\lambda(r)+\alpha(r))-\frac{\sigma^{2}(r)}{2}\left(\frac{f(S, I, R)}{I}\right)^{2}\right] \mathrm{d} t } \\
& +\sigma(r) \frac{f(S, I, R)}{I} \mathrm{~d} M_{B}(t) \\
= & \Gamma\left(\frac{f(S, I, R)}{I}\right) \mathrm{d} t+\sigma(r) \frac{f(S, I, R)}{I} \mathrm{~d} M_{B}(t),
\end{aligned}
$$

with $\Gamma(z)=-(\mu(i)+\lambda(i)+\alpha(i))+\beta(i) z-\frac{\sigma^{2}(i)}{2} z^{2}$, for $i \in \mathbb{I}$. Note that, for any $i \in \mathbb{I}$ the function $\Gamma$ in monotone increasing for $z \in\left[0, \beta(i) / \sigma^{2}(i)\right]$. Using the condition $\sigma^{2} \leq \beta / \partial f\left(\overline{m_{S}}, 0,0\right) / \partial I$ and the fact that $\frac{f(S, I, R)}{I} \leq \frac{\partial f\left(\overline{m_{S}}, 0,0\right)}{\partial I}$, we have

$$
\Gamma(z) \leq \Gamma\left(\frac{\partial f\left(\overline{m_{S}}, 0,0\right)}{\partial I}\right)
$$


Then,

$$
\begin{aligned}
d \ln I(t)= & {\left[\beta(r) \frac{\partial f\left(\overline{m_{S}}, 0,0\right)}{\partial I}-(\mu(r)+\lambda(r)+\alpha(r))-\frac{\sigma^{2}(r)}{2}\left(\frac{\partial f\left(\overline{m_{S}}, 0,0\right)}{\partial I}\right)^{2}\right] \mathrm{d} t } \\
& +\sigma(r) \frac{f(S, I, R)}{I} \mathrm{~d} M_{B}(t) \\
\triangleq & \bar{R}(r) \mathrm{d} t+\sigma(r) \frac{f(S, I, R)}{I} \mathrm{~d} M_{B}(t) .
\end{aligned}
$$

Integrating both sides of (2.1) from 0 to $t$ and then dividing by $t$, we get

$$
\frac{\ln I(t)}{t}=\frac{\ln I(0)}{t}+\frac{1}{t} \int_{0}^{t} \bar{R}(r(\tau)) \mathrm{d} \tau+\frac{1}{t} \int_{0}^{t} \sigma(r(\tau)) \frac{f(S(\tau), I(\tau), R(\tau))}{I(\tau)} \mathrm{d} M_{B}(\tau)
$$

Using the ergodicity of Markov chain $r(t)$, we have

$$
\limsup _{t \rightarrow \infty} \frac{1}{t} \int_{0}^{t} \bar{R}(r(\tau)) \mathrm{d} \tau=\sum_{i=0}^{N} \pi_{i} \bar{R}(i)
$$

Taking the superior limit on both sides of (2.2), using the strong law of large numbers for local martingales and (2.3), we obtain

$$
\begin{aligned}
\limsup _{t \rightarrow \infty} \frac{\ln I(t)}{t} & \leq \sum_{i=0}^{N} \pi_{i} \bar{R}(i) \\
& \triangleq \sum_{i=0}^{N} \pi_{i}\left[\mu(i)+\lambda(i)+\alpha(i)+\frac{\sigma^{2}(i)}{2}\left(\frac{\partial f\left(\overline{m_{S}}, 0,0\right)}{\partial I}\right)^{2}\right]\left[\mathcal{R}_{\text {swt }}-1\right], \text { a.s. }
\end{aligned}
$$

Since $\mathcal{R}_{\text {swt }}<1$, we conclude that

$$
\limsup _{t \rightarrow \infty} \frac{\ln I(t)}{t}<0, \text { a.s. }
$$

which implies that

$$
\lim _{t \rightarrow \infty} I(t)=0, \text { a.s. }
$$

Therefore, let $\bar{\Theta}=\left\{\omega \in \Omega: \lim _{t \rightarrow \infty} I(t)=0\right\}$, then (2.4) implies $\mathbb{P}(\bar{\Theta})=1$. Hence, for any $\omega \in \bar{\Theta}$ and any constant $\varepsilon>0$, there exists a constant $C_{\varepsilon}^{\omega}>0$ such that

$$
I^{\omega}(t) \leq \varepsilon, \text { for all } t>C_{\varepsilon}^{\omega} .
$$

From (2.5) and the third equation of system (1.2), we obtain

$$
\mathrm{d} R^{\omega}(t) \leq\left[\check{\gamma} I^{\omega}(t)-(\hat{\mu}+\hat{\delta}) R^{\omega}(t)\right] \mathrm{d} t
$$




$$
\leq\left[\check{\gamma} \varepsilon-(\hat{\mu}+\hat{\delta}) R^{\omega}(t)\right] \mathrm{d} t
$$

for all $\omega \in \bar{\Theta}, t>C_{\varepsilon}^{\omega}$.

Therefore, using the comparison theorem we get

$$
\limsup _{t \rightarrow \infty} R^{\omega}(t) \leq \frac{\check{\gamma} \varepsilon}{\hat{\mu}+\hat{\delta}}, \text { for all } \omega \in \bar{\Theta} \text {. }
$$

We see that $R^{\omega}(t)>0$ for all $\omega$ in $\bar{\Theta}$ and $t>0$, by arbitrariness of $\varepsilon$, we get

$$
\lim _{t \rightarrow \infty} R^{\omega}(t)=0, \omega \in \bar{\Theta} .
$$

We have $\mathbb{P}(\bar{\Theta})=1$, thus we get

$$
\lim _{t \rightarrow \infty} R(t)=0, \text { a.s. }
$$

This completes the proof.

Remark 2.2. Noting that the number $R_{s w t}$ is smaller than $R_{0}$. This means that the disease in the stochastic model (1.2) with Markov switch will go extinct with probability one but the disease in the corresponding deterministic model persists.

\section{Persistence of the disease}

In the following, we propose sufficient conditions for the persistence in mean of disease in model (1.2). Then, we have the following result.

Theorem 3.1. Assume that $\left(\boldsymbol{A} \boldsymbol{s}_{1}\right)$ and $\left(\boldsymbol{A} s_{2}\right)$ hold. If $R_{\text {swt }}>1$, then for any given initial value $(S(0), I(0), R(0)) \in \Lambda$, the solution of (1.2) satisfies

$$
\begin{aligned}
& \liminf _{t \rightarrow \infty} \frac{1}{t} \int_{0}^{t} S(s) \mathrm{d} s \geq W_{3}\left[\mathcal{R}_{s w t}-1\right] \text { a.s. } \\
& \liminf _{t \rightarrow \infty} \frac{1}{t} \int_{0}^{t} I(s) \mathrm{d} s \geq W_{1}\left[\mathcal{R}_{s w t}-1\right] \text { a.s. } \\
& \liminf _{t \rightarrow \infty} \frac{1}{t} \int_{0}^{t} R(s) \mathrm{d} s \geq W_{2}\left[\mathcal{R}_{s w t}-1\right] \quad a . s .
\end{aligned}
$$

where $W_{i},(i=1,2,3)$ are positive constants.

Proof. First, applying the generalized Itô's formula on the function $\Psi(I, i)=\ln I+\xi(i)$, we have

$$
\begin{aligned}
d \Psi= & {\left[\beta(i) \frac{f(S, I, R)}{I}-(\mu(i)+\lambda(i)+\alpha(i))-\frac{\sigma^{2}(i)}{2}\left(\frac{f(S, I, R)}{I}\right)^{2}\right.} \\
& \left.+\sum_{k=1}^{N} \phi_{i k} \xi(k)\right] \mathrm{d} t+\sigma(i) \frac{f(S, I, R)}{I} \mathrm{~d} M_{B}(t) .
\end{aligned}
$$


From $\left(\mathbf{A} \mathbf{s}_{1}\right)$, we get

$$
\begin{aligned}
d \Psi \geq & {\left[\beta(i) \frac{f(S, I, R)}{I}-(\mu(i)+\lambda(i)+\alpha(i))-\frac{\sigma^{2}(i)}{2}\left(\frac{\partial f\left(\overline{m_{S}}, 0,0\right)}{\partial I}\right)^{2}\right.} \\
& \left.+\sum_{k=1}^{N} \phi_{i k} \xi(k)\right] \mathrm{d} t+\sigma(i) \frac{f(S, I, R)}{I} \mathrm{~d} M_{B}(t),
\end{aligned}
$$

then

$$
\begin{aligned}
d \Psi \geq & {\left[\beta(i) \frac{\partial f\left(\overline{m_{S}}, 0,0\right)}{\partial I}-(\mu(i)+\lambda(i)+\alpha(i))-\frac{\sigma^{2}(i)}{2}\left(\frac{\partial f\left(\overline{m_{S}}, 0,0\right)}{\partial I}\right)^{2}\right.} \\
& \left.+\sum_{k=1}^{N} \phi_{i k} \xi(k)\right] \mathrm{d} t+\beta(i)\left(\frac{f(S, I, R)}{I}-\frac{f\left(\overline{m_{S}}, I, R\right)}{I}+\frac{f\left(\overline{m_{S}}, I, R\right)}{I}\right. \\
& \left.-\frac{\partial f\left(\overline{m_{S}}, 0, R\right)}{\partial I}+\frac{\partial f\left(\overline{m_{S}}, 0, R\right)}{\partial I}-\frac{\partial f\left(\overline{m_{S}}, 0,0\right)}{\partial I}\right) \mathrm{d} t+\sigma(i) \frac{f(S, I, R)}{I} \mathrm{~d} M_{B}(t) .
\end{aligned}
$$

By the Lagrange's mean value theorem, we get

$$
\begin{aligned}
& \frac{f(S(t), I(t), R(t))}{I(t)}-\frac{f\left(\overline{m_{S}}, I(t), R(t)\right)}{I(t)}+\frac{f\left(\overline{m_{S}}, I(t), R(t)\right)}{I(t)}-\frac{\partial f\left(\overline{m_{S}}, 0, R(t)\right)}{\partial I} \\
& +\frac{\partial f\left(\overline{m_{S}}, 0, R(t)\right)}{\partial I}-\frac{\partial f\left(\overline{m_{S}}, 0,0\right)}{\partial I} \\
& =\frac{1}{I(t)} \frac{\partial f\left(\varpi_{1}(t), I(t), R(t)\right)}{\partial S}\left(S(t)-\overline{m_{S}}\right)+\left[\frac{1}{\varpi_{2}(t)} \frac{\partial f\left(\overline{m_{S}}, \varpi_{2}(t), R(t)\right)}{\partial I}\right. \\
& \left.-\frac{f\left(\overline{m_{S}}, \varpi_{2}(t), R(t)\right)}{\varpi_{2}^{2}(t)}\right] I(t)+\frac{\partial^{2} f\left(\overline{m_{S}}, 0, \varpi_{3}(t)\right)}{\partial I \partial R} R(t),
\end{aligned}
$$

with $\varpi_{1}(t) \in\left(S(t), \overline{m_{S}}\right), \varpi_{2}(t) \in(0, I(t))$ and $\varpi_{3}(t) \in(0, R(t))$. Then

$$
\begin{aligned}
d \Psi \geq & {\left[\beta(i) \frac{\partial f\left(\overline{m_{S}}, 0,0\right)}{\partial I}-(\mu(i)+\lambda(i)+\alpha(i))-\frac{\sigma^{2}(i)}{2}\left(\frac{\partial f\left(\overline{m_{S}}, 0,0\right)}{\partial I}\right)^{2}\right.} \\
& +\sum_{k=1}^{N} \phi_{i k} \xi(k)+\beta(i) \frac{1}{I(t)} \times \frac{\partial f\left(\varpi_{1}(t), I(t), R(t)\right)}{\partial S}\left(S(t)-\overline{m_{S}}\right) \\
& +\beta(i) \frac{\frac{\partial f\left(\overline{m_{S}}, \varpi_{2}(t), R(t)\right)}{\partial I} \varpi_{2}(t)-f\left(\overline{m_{S}}, \varpi_{2}(t), R(t)\right)}{\varpi_{2}^{2}(t)} I(t) \\
& \left.+\beta(i) \frac{\partial^{2} f\left(\overline{m_{S}}, 0, \varpi_{3}(t)\right)}{\partial I \partial R} R(t)\right] \mathrm{d} t+\sigma(i) \frac{f(S, I, R)}{I} \mathrm{~d} M_{B}(t) .
\end{aligned}
$$

By virtue of Lemma 1.2 , since $\left(\varpi_{1}(t), I(t), R(t)\right) \in \Lambda,\left(\overline{m_{S}}, \varpi_{2}(t), R(t)\right) \in \Lambda$ and $\left(\overline{m_{S}}, 0, \varpi_{3}(t)\right) \in \Lambda$ a.s. for all $t \geq 0$, we have

$$
\frac{1}{I(t)} \frac{\partial f\left(\varpi_{1}(t), I(t), R(t)\right)}{\partial S} \leq C_{1}, \frac{\partial^{2} f\left(\overline{m_{S}}, 0, \varpi_{3}(t)\right)}{\partial I \partial R} \geq-C_{2} \text { a.s. }
$$




$$
\frac{\frac{\partial f\left(\bar{m}_{S}, \varpi_{2}(t), R(t)\right)}{\partial I} \varpi_{2}(t)-f\left(\overline{m_{S}}, \varpi_{2}(t), R(t)\right)}{\varpi_{2}^{2}(t)} \geq-C_{3} \text { a.s. }
$$

where

$$
\begin{gathered}
C_{1}=\max \left\{\left|\frac{1}{I} \frac{\partial f(S, I, R)}{\partial S}\right|\right\}<\infty, \quad C_{2}=\max \left\{\left|\frac{\partial^{2} f\left(\overline{m_{S}}, 0, R\right)}{\partial I \partial R}\right|\right\}<\infty, \\
C_{3}=\max \left\{\left|\frac{\frac{\partial f\left(\overline{m_{S}}, I, R\right)}{\partial I} \varpi_{2}(t)-f\left(\overline{m_{S}}, I, R\right)}{\varpi_{2}^{2}(t)}\right|\right\}<\infty .
\end{gathered}
$$

From the inequalities above we obtain

$$
\begin{aligned}
d \Psi \geq & {\left[\beta(i) \frac{\partial f\left(\overline{m_{S}}, 0,0\right)}{\partial I}-(\mu(i)+\lambda(i)+\alpha(i))-\frac{\sigma^{2}(i)}{2}\left(\frac{\partial f\left(\overline{m_{S}}, 0,0\right)}{\partial I}\right)^{2}\right.} \\
& \left.+\sum_{k=1}^{N} \phi_{i k} \xi(k)\right] \mathrm{d} t-\check{\beta}\left[C_{1}\left(\overline{m_{S}}-S\right)+C_{2} I+C_{3} R\right] \mathrm{d} t \\
& +\sigma(i) \frac{f(S, I, R)}{I} \mathrm{~d} M_{B}(t) \\
= & {\left[\bar{R}^{*}(i)+\sum_{k=1}^{N} \phi_{i k} \xi(k)\right] \mathrm{d} t-\check{\beta}\left[C_{1}\left(\overline{m_{S}}-S\right)+C_{2} I+C_{3} R\right] \mathrm{d} t } \\
& +\sigma(i) \frac{f(S, I, R)}{I} \mathrm{~d} M_{B}(t) .
\end{aligned}
$$

Since the generator matrix $\Phi$ is irreducible, for $\bar{R}^{*}=\left(\bar{R}^{*}(1), \bar{R}^{*}(2), \ldots, \bar{R}^{*}(N)\right)$ such that $\bar{R}^{*}(i)=$ $\beta(i) \frac{\partial f\left(\overline{m_{S}}, 0,0\right)}{\partial I}-(\mu(i)+\lambda(i)+\alpha(i))-\frac{\sigma^{2}(i)}{2}\left(\frac{\partial f\left(\overline{m_{S}}, 0,0\right)}{\partial I}\right)^{2}$, there exists $\xi=(\xi(1), \xi(2), \ldots, \xi(N))$ solution of the Poisson system

$$
\Phi \xi=-\bar{R}^{*}+\sum_{i=1}^{N} \pi_{i} \bar{R}^{*}(i)\left(\begin{array}{c}
1 \\
1 \\
\cdot \\
\cdot \\
1
\end{array}\right)
$$

Then,

$$
\begin{aligned}
\bar{R}^{*}(i)+\sum_{k=1}^{N} \phi_{i k} \xi(k) & =\sum_{i=1}^{N} \pi_{i} \bar{R}^{*}(i) \\
& =\sum_{i=0}^{N} \pi_{i}\left[\mu(i)+\lambda(i)+\alpha(i)+\frac{\sigma^{2}(i)}{2}\left(\frac{\partial f\left(\overline{m_{S}}, 0,0\right)}{\partial I}\right)^{2}\right]\left[\mathcal{R}_{s w t}-1\right] .
\end{aligned}
$$


On the other hand from the system (1.2), we have

$$
\begin{aligned}
\mathrm{d} S+\mathrm{d} I+\frac{\hat{\delta}}{\check{\mu}+\check{\delta}} \mathrm{d} R & =\left[A(r)-\mu(r) S-\left(\mu(r)+\alpha(r)+\gamma(r)-\frac{\hat{\delta} \gamma(r)}{\check{\mu}+\check{\delta}}\right) I\right] \mathrm{d} t \\
& \geq\left[\hat{A}-\check{\mu} S-\left(\check{\mu}+\check{\alpha}+\check{\gamma}-\frac{\hat{\delta} \hat{\gamma}}{\check{\mu}+\check{\delta}}\right) I\right] \mathrm{d} t
\end{aligned}
$$

integrating from 0 to $t$ and dividing by $t$, we obtain

$$
\begin{aligned}
& \frac{S(t)+I(t)}{t}-\frac{S(0)+I(0)}{t}+\frac{\hat{\delta}}{\check{\mu}+\check{\delta}} \frac{R(t)-R(0)}{t} \\
\geq & \hat{A}-\check{\mu} \frac{1}{t} \int_{0}^{t} S(s) \mathrm{d} s-\left(\check{\mu}+\check{\alpha}+\check{\gamma}-\frac{\hat{\delta} \hat{\gamma}}{\check{\mu}+\check{\delta}}\right) \frac{1}{t} \int_{0}^{t} I(s) \mathrm{d} s,
\end{aligned}
$$

Therefore,

$$
-\left(\overline{m_{S}}-\frac{1}{t} \int_{0}^{t} S(s) \mathrm{d} s\right) \geq-\frac{\check{A}}{\hat{A} \hat{\mu}}\left(\check{\mu}+\check{\alpha}+\check{\gamma}-\frac{\hat{\delta} \hat{\gamma}}{\check{\mu}+\check{\delta}}\right) \frac{1}{t} \int_{0}^{t} I(s) \mathrm{d} s-\frac{\check{A} \chi(t)}{\hat{A} \hat{\mu}},
$$

where $\chi(t)=\frac{S(t)+I(t)}{t}-\frac{S(0)+I(0)}{t}+\frac{\hat{\delta}}{\breve{\mu}+\check{\delta}} \frac{R(t)-R(0)}{t}$. And we have

$$
\frac{1}{t} \int_{0}^{t} R(s) \mathrm{d} s \leq \frac{\check{\gamma}}{\hat{\mu}+\hat{\delta}} \frac{1}{t} \int_{0}^{t} I(s) \mathrm{d} s-\frac{R(t)-R(0)}{(\hat{\mu}+\hat{\delta}) t}
$$

An integration on both sides of (3.1) and dividing by $t$, from (3.2) and (3.3) we obtain

$$
\begin{aligned}
\frac{\Psi(t)-\Psi(0)}{t} \geq & \sum_{i=0}^{N} \pi_{i}\left[\mu(i)+\lambda(i)+\alpha(i)+\frac{\sigma^{2}(i)}{2}\left(\frac{\partial f\left(\overline{m_{S}}, 0,0\right)}{\partial I}\right)^{2}\right]\left[\mathcal{R}_{s w t}-1\right] \\
& -\check{\beta} C_{1} \frac{\check{A}}{\hat{A} \hat{\mu}}\left(\check{\mu}+\check{\alpha}+\check{\gamma}-\frac{\hat{\delta} \hat{\gamma}}{\check{\mu}+\check{\delta}}\right) \frac{1}{t} \int_{0}^{t} I(s) \mathrm{d} s-\check{\beta} C_{1} \frac{\check{A}}{\hat{A} \hat{\mu}} \chi(t) \\
& -\check{\beta} C_{2} \frac{1}{t} \int_{0}^{t} I(s) \mathrm{d} s-\check{\beta} C_{3} \frac{\check{\gamma}}{\hat{\mu}+\hat{\delta}} \frac{1}{t} \int_{0}^{t} I(s) \mathrm{d} s+\check{\beta} C_{3} \frac{R(t)-R(0)}{(\hat{\mu}+\hat{\delta}) t} \\
& +\frac{1}{t} \int_{0}^{t} \sigma(r(s)) \frac{f(S(s), I(s), R(s))}{I} \mathrm{~d} M_{B}(s) .
\end{aligned}
$$

This implies that

$$
\begin{aligned}
\frac{\Psi(t)-\Psi(0)}{t} \geq & \sum_{i=0}^{N} \pi_{i}\left[\mu(i)+\lambda(i)+\alpha(i)+\frac{\sigma^{2}(i)}{2}\left(\frac{\partial f\left(\overline{m_{S}}, 0,0\right)}{\partial I}\right)^{2}\right]\left[\mathcal{R}_{s w t}-1\right] \\
& -\check{\beta}\left[C_{1} \frac{\check{A}}{\hat{A} \hat{\mu}}\left(\check{\mu}+\check{\alpha}+\check{\gamma}-\frac{\hat{\delta} \hat{\gamma}}{\check{\mu}+\check{\delta}}\right)+C_{2}+C_{3} \frac{\check{\gamma}}{\hat{\mu}+\hat{\delta}}\right] \frac{1}{t} \int_{0}^{t} I(s) \mathrm{d} s+\Theta(t),
\end{aligned}
$$


where

$$
\Theta(t)=-\check{\beta} C_{1} \frac{\check{A}}{\hat{A} \hat{\mu}} \chi(t)+\check{\beta} C_{3} \frac{R(t)-R(0)}{(\hat{\mu}+\hat{\delta}) t}+\frac{1}{t} \int_{0}^{t} \sigma(r(s)) \frac{f(S(s), I(s), R(s))}{I} \mathrm{~d} M_{B}(s) .
$$

The large number theorem for local martingales and from $\left(\mathbf{A s}_{2}\right)$ we have

$$
\lim _{t \rightarrow \infty} \Theta(t)=0 \text { a.s. }
$$

Additionally, we have

$$
\lim _{t \rightarrow \infty} \frac{\Psi(t)-\Psi(0)}{t}=0, \text { a.s. }
$$

Hence

$$
\liminf _{t \rightarrow \infty} \frac{1}{t} \int_{0}^{t} I(s) \mathrm{d} s \geq W_{1}\left[\mathcal{R}_{s w t}-1\right] \text { a.s. }
$$

From the third equation of system (1.2), we can show that

$$
\frac{1}{t} \int_{0}^{t} R(s) \mathrm{d} s \geq \frac{\check{\gamma}}{\hat{\mu}+\hat{\delta}} \frac{1}{t} \int_{0}^{t} I(s) \mathrm{d} s-\frac{R(t)-R(0)}{(\hat{\mu}+\hat{\delta}) t} .
$$

As $t \rightarrow \infty$, we have

$$
\lim _{t \rightarrow \infty} \frac{R(t)-R(0)}{t}=0 \text { a.s. }
$$

Taking the inferior limit on both sides of (3.4), we obtain

$$
\begin{aligned}
\liminf _{t \rightarrow \infty} \frac{1}{t} \int_{0}^{t} R(s) \mathrm{d} s & \geq \frac{\check{\gamma} W_{1}}{\hat{\mu}+\hat{\delta}}\left[\mathcal{R}_{s w t}-1\right] \\
& \triangleq W_{2}\left[\mathcal{R}_{s w t}-1\right] .
\end{aligned}
$$

Similarly, we can show that

$$
\begin{aligned}
\liminf _{t \rightarrow \infty} \frac{1}{t} \int_{0}^{t} S(s) \mathrm{d} s & \geq \frac{\hat{A}+\hat{\delta} W_{2}}{\check{\mu}+\check{\beta} Q_{S}}\left[\mathcal{R}_{s w t}-1\right] \\
& \triangleq W_{3}\left[\mathcal{R}_{s w t}-1\right]
\end{aligned}
$$

where $Q_{S}=\max _{\Lambda}\left\{\frac{f(S, I, R)}{S}\right\}$.

\section{Applichtions}

Using the Milsteins Higher Order Method given in [10], the analytical contributions for the stochastic model described in this paper are confirmed numerically. Next, we assume $r(t)$ is a right-continuous Markov chain 

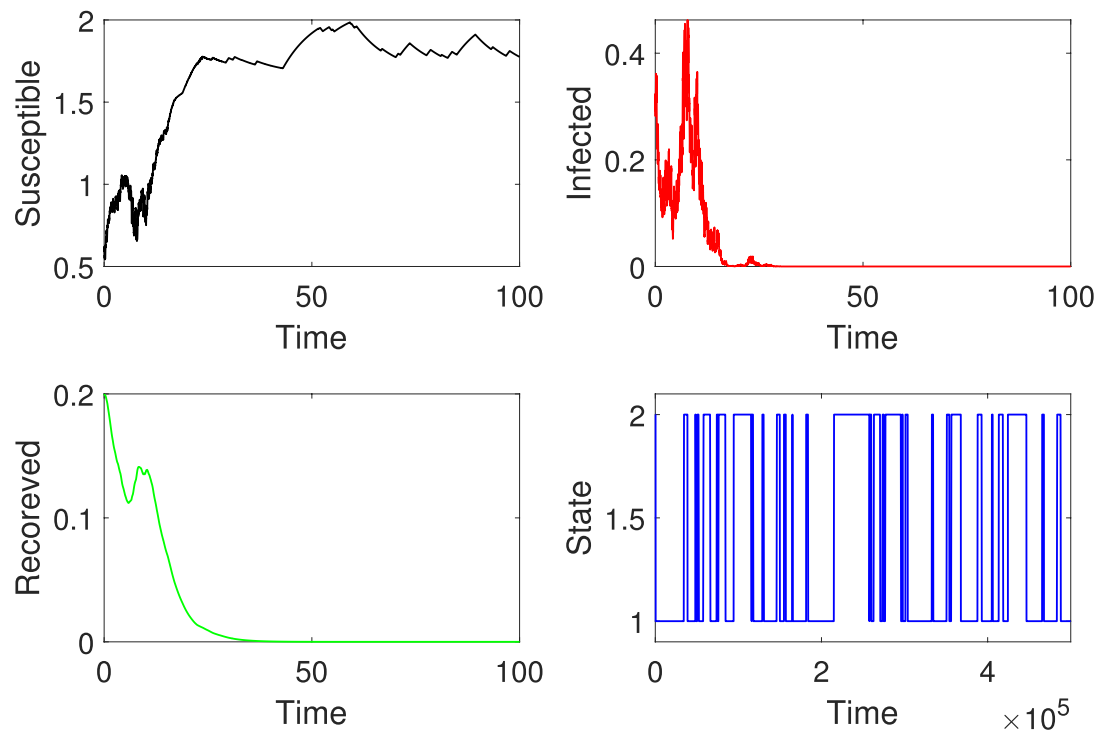

Figure 1. Trajectories of stochastic system for case I, and Markov chain $r(t)$.

taking values in a finite state space $\mathbb{I}=\{1,2\}$ with the generator

$$
\Phi=\left(\begin{array}{cc}
-1 & 1 \\
2 & -2
\end{array}\right)
$$

Then the stationary distribution of $r(t)$ is displayed by

$$
\pi=\left(\pi_{1}, \pi_{2}\right)=(2 / 3,1 / 3) .
$$

We consider the following nonlinear incidence rate satisfied the assumptions in this paper

$$
f(S, I, R)=\frac{\beta S I}{1+\alpha_{1} S+\alpha_{2} I+\alpha_{3} S I},
$$

where $\alpha_{1}, \alpha_{2}, \alpha_{3}$ are constants. Notice that the incidence above combines many general forms existing in the literature (see, $[4,5,11,27,34])$. Then, we let the parameter values in model (1.2) as follows:

$A(1)=0.2, \quad A(2)=0.25, \quad \beta(1)=0.54, \quad \beta(2)=0.56, \quad \mu(1)=0.12, \quad \mu(2)=0.12, \quad \gamma(1)=0.13, \quad \gamma(2)=$ $0.13, \alpha(1)=0.11, \alpha(2)=0.21, \delta(1)=0.11, \delta(2)=0.13, \quad \alpha_{1}(1)=0.1, \alpha_{1}(2)=0.1, \alpha_{2}(1)=0.1, \alpha_{2}(2)=$ $0.1, \alpha_{3}(1)=0.1, \alpha_{3}(2)=0.1$

Also, we assume that the initial value of model (1.2) is $(0.6,0.3,0.2) \in \Lambda \times \mathbb{I}$. Then, let's treat the following two illustrations cases of simulation

(I) In this case we choose $\sigma(1)=0.77, \sigma(2)=0.75$. Simple compilation leads to $\mathcal{R}_{\text {swt }}=0.4016$. By Theorem 2.1. we conclude that the disease in model (1.2) goes extinct. The computer simulation given in Figure 1. shown this result.

(II) We choose the white noise values such that $\sigma(1)=0.27, \sigma(2)=0.25$. By computing, we find that $\mathcal{R}_{\text {swt }}=1.0706>1$. From the computers simulations given in Figure 2, we can see that the diseases in model (1.2) 

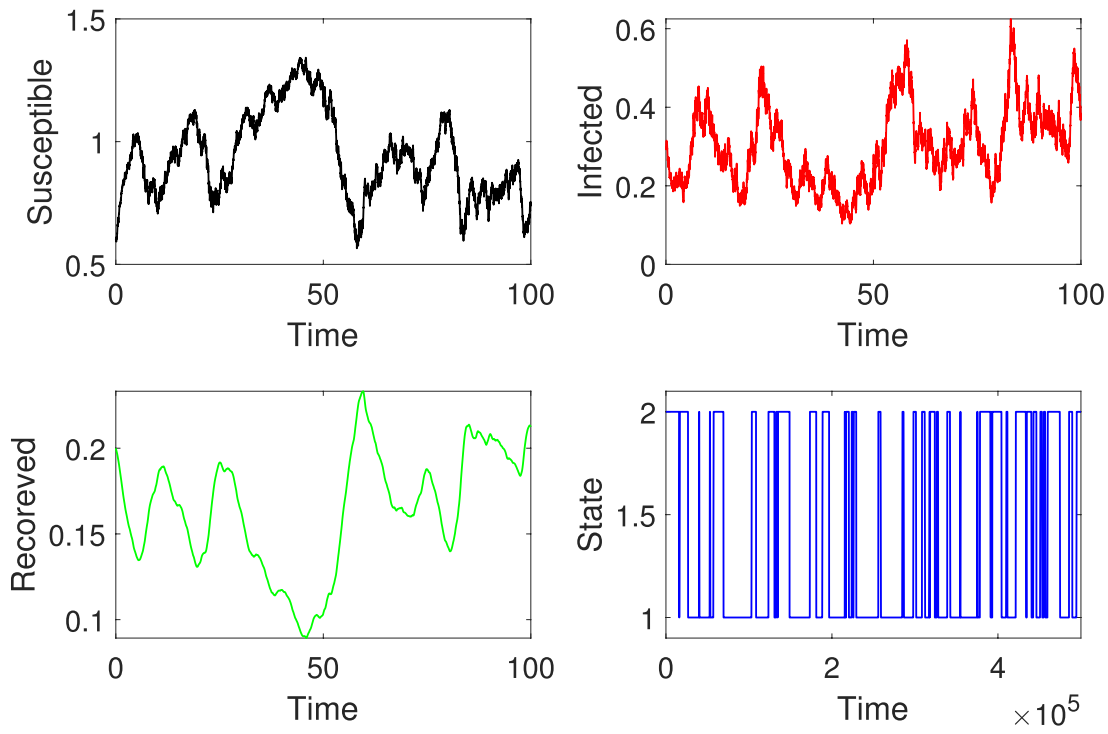

FIgURE 2. Trajectories of stochastic system for case II, and Markov chain $r(t)$.

persist in the human population. Therefore, according to the cases above the large intensity of white noise will complete the disease towards extinction.

\section{Conclusion}

The spreading of infectious disease presents a more big problem that involves a high loss of economies of many countries. In addition, a large number of individuals in the world die from infectious diseases. For these reasons, many specialists have developed mathematical models to interpret and proposed the directions for decreasing the epidemic diffusion. Firstly, many authors were interested in the proposition of deterministic models. But, these models presented some limitations due to the ignoring of environmental fluctuations. Therefore, a new generation of epidemic models integrating the randomness properties that describe the manners of distribution of infectious diseases has developed.

The present paper investigates a class of stochastic switched SIRS epidemic model with general nonlinear incidence. Using the stochastic Lyapunov approach, sufficient conditions are established to guarantee the extinction or persistence of epidemic disease by introducing an appropriate stochastic threshold value $\mathcal{R}_{s w t}$. Precisely, we obtain the following result:

- Let assumptions $\left(\mathbf{A} \mathbf{s}_{1}\right)$ and $\left(\mathbf{A} \mathbf{s}_{2}\right)$ hold, if

$$
\mathcal{R}_{s w t}=\frac{\sum_{i=0}^{N} \pi_{i} \beta(i) \frac{\partial f\left(\overline{m_{S}}, 0,0\right)}{\partial I}}{\sum_{i=0}^{N} \pi_{i}\left[\mu(i)+\lambda(i)+\alpha(i)+\frac{\sigma^{2}(i)}{2}\left(\frac{\partial f\left(\overline{m_{S}}, 0,0\right)}{\partial I}\right)^{2}\right]}<1,
$$

then epidemic infection dies out with probability one, if $\mathcal{R}_{s w t}>1$ the epidemic disease persists in the population.

This shows that the large white noise is beneficial to control infectious disease. The stochastic system (1.2) has not taken into account the impact of some brusque and sudden phenomena as tsunami, earthquakes, pandemic, etc., to taken into account these phenomena, we propose to apply a jumps process on the model (1.2) in our future work. 


\section{REFERENCES}

[1] J.R. Beddington, Mutual interference between parasites or predators and its effect on searching efficiency. J. Anim. Ecol. 44 (1975) 331-340.

[2] Z. Chen, Z. Tian, S. Zhang and C. Wei, The stationary distribution and ergodicity of a stochastic phytoplankton-zooplankton model with toxin-producing phytoplankton under regime switching. Physica A 537 (2020) 122728.

[3] N.T. Dieu, T. Fugo and N.H. Du, Asymptotic behaviors of stochastic epidemic models with jump-diffusion. Appl. Math. Model. 86 (2020) 259-270.

[4] N.H. Du, N.T. Dieu and N.N. Nhu, Conditions for permanence and ergodicity of certain SIR epidemic models. Acta Appl. Math. 160 (2019) 81-99.

[5] A. El Koufi, A. Bennar, N. El Koufi and N. Yousfi, Asymptotic properties of a stochastic SIQR epidemic model with Lévy Jumps and Beddington-DeAngelis incidence rate. Results Phys. 27 (2021) 104472.

[6] A. El Koufi, J. Adnani, A. Bennar and N. Yousfi, Analysis of a stochastic SIR model with vaccination and nonlinear incidence rate. Int. J. Diff. Equat. 2019 (2019).

[7] Z. Han and J. Zhao, Stochastic SIRS model under regime switching. Nonlinear Anal-Real. 14 (2013) $352-364$.

[8] P. Han, Z. Chang and X. Meng, Asymptotic dynamics of a stochastic sir epidemic system affected by mixed nonlinear incidence rates. Complexity 2020 (2020).

[9] H. Hethcote, M. Zhien and L. Shengbing, Effects of quarantine in six endemic models for infectious diseases. Math. Biosci. 180 (2002) 141-160.

[10] D.J. Higham, An algorithmic introduction to numerical simulation of stochastic differential equations. SIAM Rev. 43 (2001) $525-546$.

[11] L. Huo, J. Jiang, S. Gong and B. He, Dynamical behavior of a rumor transmission model with Holling-type II functional response in emergency event. Physica A 450 (2016) 228-240.

[12] M.N. Jan, N. Ali, G. Zaman, I. Ahmad, Z. Shah and P. Kumam, HIV-1 infection dynamics and optimal control with CrowleyMartin function response. Comput. Meth. Prog. Biol. 193 (2020) 105503.

[13] D. Jiang, J. Yu, C. Ji and N. Shi, Asymptotic behavior of global positive solution to a stochastic SIR model. Math. Comput. Model. 54 (2011) 221-232.

[14] W.O. Kermack and A.G. McKendrick, Contributions to the mathematical theory of epidemics (part I). Proc. R. Soc. Lond. Ser. A 115 (1927) 700-721.

[15] A.E. Koufi, A. Bennar and N. Yousfi, Dynamics of a stochastic SIRS epidemic model with regime switching and specific functional response. Discr. Dyn. Nat. Soc. 2020 (2020).

[16] M.A. Khan, Y. Khan and S. Islam, Complex dynamics of an SEIR epidemic model with saturated incidence rate and treatment. Physica A 493 (2018) 210-227.

[17] R. Khasminskii, Stochastic stability of differential equations. Springer Science Business Media (2011).

[18] G. Lan, Z. Lin, C. Wei and S. Zhang, A stochastic SIRS epidemic model with non-monotone incidence rate under regimeswitching. J. Frankl. Inst. 356 (2019) 9844-9866.

[19] G. Lan, Z. Lin, C. Wei and S. Zhang, A stochastic SIRS epidemic model with non-monotone incidence rate under regimeswitching. J. Frankl. Inst. 356 (2019) 9844-9866.

[20] Y. Lin, L. Wang and X. Dong, Long-time behavior of a regime-switching SIRS epidemic model with degenerate diffusion. Physica A 529 (2019) 121551.

[21] T. Li, F. Zhang, H. Liu and Y. Chen, Threshold dynamics of an SIRS model with nonlinear incidence rate and transfer from infectious to susceptible. Appl. Math. Lett. 70 (2017) 52-57.

[22] Y. Liu, Y. Zhang and Q. Wang, A stochastic SIR epidemic model with Lévy jump and media coverage. Adv. Differ. Equ-Ny. 2020 (2020) 1-15.

[23] Q. Liu and D. Jiang, Threshold behavior in a stochastic SIR epidemic model with Logistic birth. Physica A 540 (2020) 123488.

[24] Q. Liu, D. Jiang, T. Hayat, A. Alsaedi and B. Ahmad, A stochastic SIRS epidemic model with logistic growth and general nonlinear incidence rate. Physica A 551 (2020) 124-152.

[25] X. Mao and C. Yuan, Stochastic differential equations with Markovian switching. Imperial College Press (2006).

[26] X. Mao, G. Marion and E. Renshaw, Environmental noise suppresses explosion in population dynamics. Stoch. Process. Appl. 97 (2002) 95-110.

[27] D. Nguyen, N. Nguyen and G. Yin, Analysis of a spatially inhomogeneous stochastic partial differential equation epidemic model. J. Appl. Probab. 57 (2020) 613-636.

[28] N.D. Phu, D. O'Regan and T.D. Tuong, Longtime characterization for the general stochastic epidemic SIS model under regime-switching. Nonlinear. Anal-Hybri. 38 (2020) 100951.

[29] N.D. Phu, D. O'Regan and T.D. Tuong, Longtime characterization for the general stochastic epidemic SIS model under regime-switching. Nonlinear. Anal-Hybri. 38 (2020) 100951.

[30] M. Pitchaimani, Stochastic dynamical probes in a triple delayed SICR model with general incidence rate and immunization strategies. Chaos. Soliton. Fractals 143 (2021) 110540.

[31] S.P. Rajasekar, M. Pitchaimani and Q. Zhu, Progressive dynamics of a stochastic epidemic model with logistic growth and saturated treatment. Physica A 538 (2020) 122649.

[32] R. Rifhat, A. Muhammadhaji and Z. Teng, Asymptotic properties of a stochastic SIRS epidemic model with nonlinear incidence and varying population sizes. Dyn. Syst. 35 (2020) 56-80. 
[33] R. Rifhat, L. Wang and Z. Teng, Dynamics for a class of stochastic SIS epidemic models with nonlinear incidence and periodic coefficients. Physica A 481 (2017) 176-190.

[34] S. Ruan and W. Wang, Dynamical behavior of an epidemic model with a nonlinear incidence rate. J. Differ. Equ. 188 (2003) $135-163$.

[35] T. Tang, Z. Teng and Z. Li, Threshold behavior in a class of stochastic SIRS epidemic models with nonlinear incidence. Stoch. Anal. Appl. 33 (2015) 994-1019.

[36] T.D. Tuong, D.H. Nguyen, N.T. Dieu and K. Tran, Extinction and permanence in a stochastic SIRS model in regime-switching with general incidence rate. Nonlinear. Anal-Hybri. 34 (2019) 121-130.

[37] C. Xu and X. Li, The threshold of a stochastic delayed SIRS epidemic model with temporary immunity and vaccination. Chaos. Solitons. Fract. 111 (2018) 227-234.

[38] Q. Yan, Y. Tang, D. Yan, J. Wang, L. Yang, X. Yang and S. Tang, Impact of media reports on the early spread of COVID-19 epidemic. J. Theor. Biol. 502 (2020) 110385.

[39] X. Yu, S. Yuan and T. Zhang, Persistence and ergodicity of a stochastic single species model with Allee effect under regime switching. Commun. Nonlinear. Sci. Numer. Simulat. 59 (2018) 359-374.

[40] X.B. Zhang, H.F. Huo, H. Xiang, Q. Shi and D. Li, The threshold of a stochastic SIQS epidemic model. Physica A 482 (2017) $362-374$.

[41] Y. Zhang, K. Fan, S. Gao, Y. Liu and S. Chen, Ergodic stationary distribution of a stochastic SIRS epidemic model incorporating media coverage and saturated incidence rate. Physica A 514 (2019) 671-685.

[42] X.B. Zhang and X.H. Zhang, The threshold of a deterministic and a stochastic SIQS epidemic model with varying total population size. Appl. Math. Model. 91 (2021) 749-767.

\section{Subscribe to Open (S2O) A fair and sustainable open access model}

This journal is currently published in open access under a Subscribe-to-Open model (S2O). S2O is a transformative model that aims to move subscription journals to open access. Open access is the free, immediate, online availability of research articles combined with the rights to use these articles fully in the digital environment. We are thankful to our subscribers and sponsors for making it possible to publish this journal in open access, free of charge for authors.

\section{Please help to maintain this journal in open access!}

Check that your library subscribes to the journal, or make a personal donation to the S2O programme, by contacting subscribers@edpsciences.org

More information, including a list of sponsors and a financial transparency report, available at: https://www.edpsciences.org/en/maths-s2o-programme 